\section{SAT0685 TEMPERATURE AND SMALL PARTICULATE MATTER POLLUTION ARE ASSOCIATED WITH ORGAN SPECIFIC LUPUS FLARES: A SPATIO -TEMPORAL ANALYSIS}

G. Stojan ${ }^{1}$, A. Kvit ${ }^{2}$, F. Curriero ${ }^{2}$, M. Petri ${ }^{1} .{ }^{1}$ Rheumatology, Johns Hopkins University; ${ }^{2}$ Epidemiology, Bloomberg School of Public Health, Baltimore, USA

Background: Understanding the role of environmental exposures in the development of SLE and their association with SLE activity may help identify modifiable risk factors and potential etiological mechanisms. Cluster detection is an essential tool in public health which has the goal of detecting anomalous clusters of disease cases.

Objectives: We performed a spatial-time cluster analysis of the Johns Hopkins Lupus cohort with the goal of identifying potential spatial-time clusters of SLE organ specific disease activity related to temperature changes and fine particulate matter pollution (PM2.5).

Methods: 1261 patients who fulfil 4 of the 11 American College of Rheumatology classification criteria for SLE and who had recorded home addresses were included in the analysis. Disease activity was expressed as Physician Global Estimate (PGA), and included rash, joint, serological, neural, renal, pulmonary, and haematological flare-ups. The area utilised in this analysis was a 350 kilometre radial buffer around the Johns Hopkins Lupus Centre. This area was considered due to the high and consistent density of study participants. The data ranged from January 1999 to February 2009. Average temperature and PM2.5 exposure over a period of 10 days prior to patient visit was obtained from the United States Environmental Protection Agency, and county level demographics were obtained from the US census. Univariate, multivariate, and multilevel models were built in order to study the association of these variables with lupus flare-ups. The models were adjusted for age, sex, income, racial distribution, and rural vs. urban patient residence.

Results: Rash ( $\mathrm{OR}=1.0075$ for 1 degree Fahrenheit( $\mathrm{F})$ increase), neurologic $(\mathrm{OR}=1.0096$ for 1 degree $\mathrm{F}$ increase), and joint $(\mathrm{OR}=1.011$ for 1 degree $F$ increase) flares were statistically significantly associated with an increase in temperature in univariate and multivariate analysis. Renal flares were negatively associated with increases in temperature ( $O R=0.996$ for 1 degree $F$ increase) in both univariate and multivariate analysis. Serositis flares were found to be associated in both univariate and multivariate analysis with increases in PM2.5 concentration $(\mathrm{OR}=1.024$ for an increase of $1 \mathrm{ug} / \mathrm{m} 3)$, as were hematologic flares $(\mathrm{OR}=1.019$ for an increase of $1 \mathrm{ug} / \mathrm{m} 3)$, and joint flares $(\mathrm{OR}=1.011 \mathrm{for}$ an increase of $1 \mathrm{ug} / \mathrm{m3}$ ). Maps were generated highlighting the study area and the flares. After adjusting for temperature and PM2.5, rash, neurologic, and renal flare-up clusters changed spatially and temporally, suggesting that the adjustment variables could be contributing causes to the original clusters of these kinds of flare-ups.

Conclusions: An increase in temperature was found to be significantly associated with skin, joint, and neurologic flares and inversely associated with renal flares, while increase in fine particulate matter pollution was significantly associated with serositis and hematologic flares. Spatiotemporal cluster adjustment for PM2.5 and temperature changed the neurologic, renal, and rash flare up clusters both spatially and temporally further supporting that these variables could be contributing causes to the original flare clusters. The clusters that remained unchanged indicate areas of unexplained variation that requires further study. Disclosure of Interest: None declared

DOI: 10.1136/annrheumdis-2018-eular.7338

\section{SAT0686 ARE MRI-DETECTED EROSIONS IN PATIENTS WITH UNDIFFERENTIATED ARTHRITIS PREDICTIVE FOR THE DEVELOPMENT OF RHEUMATOID ARTHRITIS? A LARGE LONGITUDINAL STUDY}

D.M. Boeters ${ }^{1}$, A.C. Boer ${ }^{1}$, A.H. van der Helm - van Mil ${ }^{1,2} .{ }^{1}$ Rheumatology, Leiden University Medical Center, Leiden; ${ }^{2}$ Rheumatology, Erasmus University Medical Center, Rotterdam, Netherlands

Background: Radiographic erosions are a clear hallmark of rheumatoid arthritis (RA) and the presence of radiographic erosions according to the EULAR definition is sufficient to classify patients as RA. Recently, the use of MRI has been recommended to detect erosions since it is more sensitive than radiography. To determine the specificity of MRI-detected erosions, we recently compared hand and foot MRI of patients presenting with RA with those of symptom-free persons and patients presenting with other arthritides. This revealed that many MRI-detected erosions were not specific for RA but a few erosion features were identified as specific for RA. These were grade $\geq 2$ erosions (defined as $>10 \%$ of bone eroded) erosions located in MTP5, and erosions located in MTP1 in persons aged $<40$. These results were derived by comparing patients that already received their final diagnosis. A clinically relevant question is whether MRI-detected erosions in patients presenting with undifferentiating arthritis $(U A)$ are valuable in predicting future progression to RA.

Objectives: To determine whether MRI-detected erosions in patients presenting with UA are valuable in predicting future progression to RA.

Methods: 302 patients consecutively presenting with UA (not fulfilling the 2010criteria) between 2010 and 2016 were studied. At baseline 1.5T MRI of the 2 nd -5 th metacarpophalangeal(MCP)- and $1 \mathrm{st}-5$ th metatarsophalangeal(MTP)joints was performed. Erosions were scored according to the RAMRIS system by 2 readers (ICC >0.93); an erosion was considered present if both readers scored $\geq 1$. First the presence of any MRI-detected erosion was evaluated. Then the presence of RA-specific erosions, defined as grade $\geq 2$ erosions, erosions in MTP5 and erosions in MTP1 in persons aged $<40$, was evaluated. Patients were followed up for 1 year on the development of RA (according to the 2010-criteria) and/or on the start of DMARDs.

Results: Of the 302 UA-patients $144(48 \%)$ developed RA and/or started DMARDs. MRI-detected erosions were observed in $57 \%$ of the 2010 UA-patients but their presence was not predictive for the development of RA (OR 1.3, $95 \% \mathrm{Cl}$ 0.8-2.0, PPV 50\%). RA-specific erosions (either a grade $\geq 2$ erosion, erosion in MTP5 and/or erosion in MTP1 in persons aged $<40$ ) were present in only $8 \%$ of the 2010 UA patients and were also not associated with RA-development (OR 0.9 $95 \% \mathrm{Cl}$ 0.4-1.9, PPV 44\%). The observed PPVs were comparable to the prior risk of $48 \%$. Similar findings were obtained when studying the individual RA-specific erosions separately.

Conclusions: MRI-detected erosions were frequently present in UA-patients. In contrast, RA-specific MRI-detected erosions were rare in UA. Since MRI-detected erosions were not associated with an increased risk on progression to RA, the present data indicate that evaluation of MRI-detected erosions is not prognostically relevant in UA-patients.

Disclosure of Interest: None declared

DOI: 10.1136/annrheumdis-2018-eular.4720

\section{SAT0687 SILICONE BREAST IMPLANTS AND THE RISK OF AUTOIMMUNE DISEASES: REAL WORLD ANALYSIS}

A. Watad ${ }^{1}$, V. Rosenberg ${ }^{2}$, S. Tiosano ${ }^{1}$, J.W. Cohen Tervaert ${ }^{3}$, Y. Yavne ${ }^{4}$, Y. Shoenfeld ${ }^{5}$, V. Shalev ${ }^{6}$, G. Chodick ${ }^{2}$, H. Amital ${ }^{4} .{ }^{1}$ Internal Medicine B, Sheba Medical Center, ${ }^{2}$ Epidemiology, Maccabi Healthcare Services, Tel-Aviv, Israel; ${ }^{3}$ Medicine and Immunology, Maastricht University, Maastricht, Netherlands; ${ }^{4}$ Sheba Medical Center, Tel-Aviv, Israel; ${ }^{5}$ The Zabludowicz Center for Autoimmune Diseases, Sheba Medical Center, ${ }^{6}$ Maccabi Healthcare Services, Tel-Aviv, Israel

Background: Previous reports have suggested an association between silicone breast implants (SBIs) and connective tissue disorders. However, several epidemiological studies have produced inconsistent results.

Objectives: To evaluate the association between SBIs and the most clinically relevant auto-immune diseases (ADs) using a large, population based database.

Methods: In this cross-sectional study, we used the computerised databases of Maccabi Healthcare Services (MHS) which include up to 20 years of data on 2 million members. Women with SBIs were identified by procedure and diagnosis codes, clinical breast examinations and mammography referrals. ADs were identified using the International Classification of Diseases 9th revision (ICD-9) codes. SBls-free women were matched by age group and socio-economic status (SES) in a ratio of 1:4. Multivariable logistic regression and Cox's proportional hazards models were performed.

Results: We included 24,651 SBI recipients and 98604 matched SBIs free women in our study. The association between SBIs and AD was significant $(p<0.05)$ (adjusted OR 1.21, 95\% Cl 1.17-1.26). The strongest association with SBIs $(O R>1.5, p<0.001)$ was recorded for systemic sclerosis (SSc) and sarcoidosis (OR of 1.99 and 1.67, respectively). Similar results were calculated when analysis was limited to cancer free women. Multivariable Cox regression model yielded a $\mathrm{HR}$ of $1.45(95 \% \mathrm{Cl} 1.21-1.73)$ for being diagnosed with at least one $A D$ in women with $\mathrm{SBI}$ compared to those without. 